\title{
A Curious Case of Idiopathic Cytopenia of Undetermined Significance (ICUS)
}

\author{
Abha Sharma, Ayush Bansal \\ Department of Medicine, ABVIMS and Dr. RML Hospital, New Delhi, India.
}

\section{Corresponding Author:}

Dr Abha Sharma

Email: abhasharma03@gmail.com

This is an Open Access article distributed under the terms of the Creative Commons Attribution License (creativecommons.org/ licenses/by/3.0).

Received Accepted Published
October 19, 2020

January 16, 2021

March 10, 2021

\begin{abstract}
Background: The term idiopathic cytopenia of undetermined significance (ICUS) is used when the probable cause of pancytopenia is not clear and it sometimes has a fatal outcome. We report this case to highlight the role of high doses of steroid in the treatment of ICUS which altered the course of disease in the patient and rendered it non-fatal. Case Report: A 28-year-old female presented with shortness of breath, pallor, icterus, ecchymosis patches, Glasgow coma scale of $8 / 15$, shock and respiratory failure. Investigations showed pancytopenia, deranged liver function test and coagulopathy. Despite being on standard treatment protocol for pancytopenia she remained in shock and had epistaxis following which a trial of high dose methylprednisolone was given and substituted with tapering dose of oral prednisone. The patient improved after this and is under 6 monthly follow-up. Conclusion: All cases of pancytopenia should be investigated thoroughly and an entity called idiopathic cytopenia of undetermined significance (ICUS) should be considered if no possible causes can be found. We share our experience of instituting steroids in a case of life threatening ICUS which benefitted the patient although the cause and mechanism were unknown.
\end{abstract}

Keywords: Blood Cells, Bone Marrow, Methylprednisolone, Pancytopenia, Prednisolone.

\section{Introduction}

The pancytopenia patient can be asymptomatic or can present with combined features of deficiency of individual cell lines such as pallor, breathlessness, susceptibility to infection, bleeding, etc [1]. The basic approach is to find out the cause of pancytopenia, which starts with a detailed history including dietary habits, socioeconomic status, drug and toxin use, followed by a thorough clinical examination with attention towards hepatosplenomegaly and lymphadenopathy [Fig.1]. Next is essential blood investigations like complete blood count, reticulocyte count, peripheral smear for type of anemia and abnormal cells [Fig.2]. Further approach is individualised depending upon history of fever, weight loss, lymph node swelling or history suggestive of autoimmune diseases then we investigate for infectious, autoimmune as well as malignant causes. The term idiopathic cytopenia

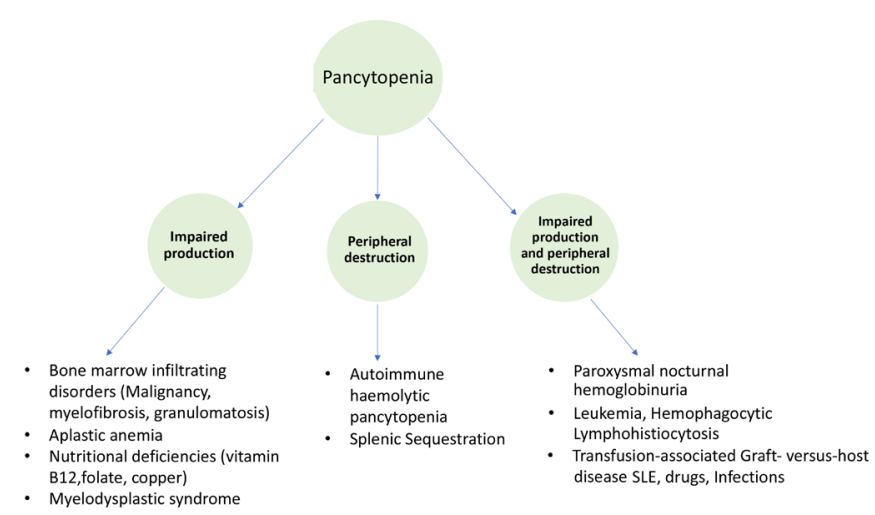

Fig.1: Causes of pancytopenia.

of undetermined significance (ICUS) is used, if even after a detailed investigation the diagnosis of the probable cause is not clear. For such patients a periodic assessment is advised to assess the evolution of the disease and to confirm or exclude a myelodysplastic syndrome (MDS). We report this case to highlight the role of high doses of steroid in the treatment of ICUS which altered the course 
of disease in the patient and rendered it non-fatal, although the mechanisms leading to this response is not known.

\section{Case Report}

A 28-year-old, unmarried female presented to the hospital with complaints of insidious onset gradually progressive shortness of breath for the past 3-4 months and altered sensorium for one day prior to admission. The patient had was no previous history of fever, night sweats, photosensitivity, headache, vomiting, joint pain, jaundice, rash, focal neurological deficit, blood transfusion, chronic diarrhoea, tuberculosis, seizure, any drug intake, menstrual abnormalities and was a vegetarian. She had an altered state of consciousness with a GCS of 8/15 (E2V2M6), shock $(\mathrm{BP}=60 / 40, P R=120 / \mathrm{min})$ and type 1 respiratory failure $\left(\mathrm{SpO}_{2}=65 \%\right)$. She had severe pallor, icterus and had ecchymosis over the abdomen and upper limbs [Fig.3] with active gum bleeding. Fundus examination showed pre-retinal hemorrhages. There was no evidence of pedal oedema, cardiac failure, hepatosplenomegaly, bony tenderness or signs of meningeal irritation.

Investigations showed pancytopenia (hemoglobin $1.3 \mathrm{~g} / \mathrm{dL}$, total leukocyte count 4140 cells $/ \mathrm{mm}^{3}$, platelets 10000 cells $/ \mathrm{mm}^{3}$ ) with macrocytic predominance (MCV $136 \mathrm{fl}$ ), reticulocyte count of 0.3 , a deranged liver function test, coagulopathy (INR 3.89), elevation of lactate dehydrogenase $(550 \mathrm{U} / \mathrm{L})$, and a peripheral smear suggestive of pancytopenia with combined deficiency anemia. Computed tomography head, ultrasound abdomen, 2-D echocardiography, vitamin B12 and folate levels were normal. Malaria serology, viral markers for hepatitis B, hepatitis C and HIV were negative. Coomb's test (direct and indirect), thyroid profile and antinuclear antibody was negative. Blood and urine cultures were normal. A provisional working diagnosis of pancytopenia with severe anemia with ischemic hepatitis with shock and hypoxic encephalopathy was considered.

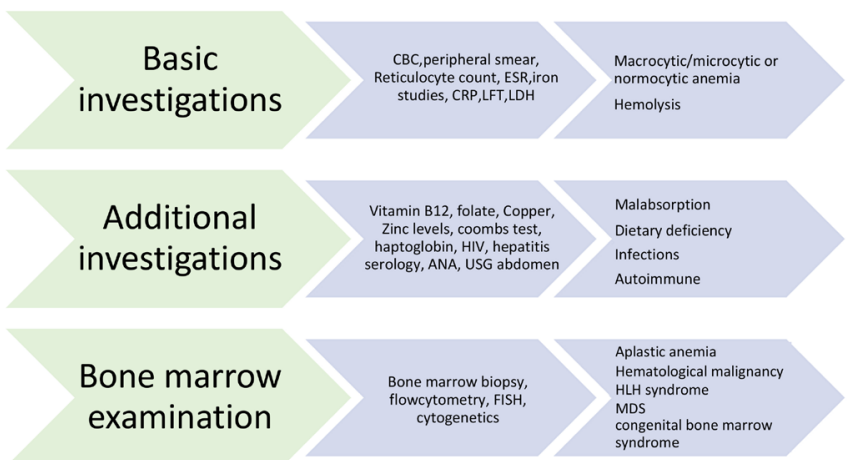

Fig.2: Investigations for pancytopenia.

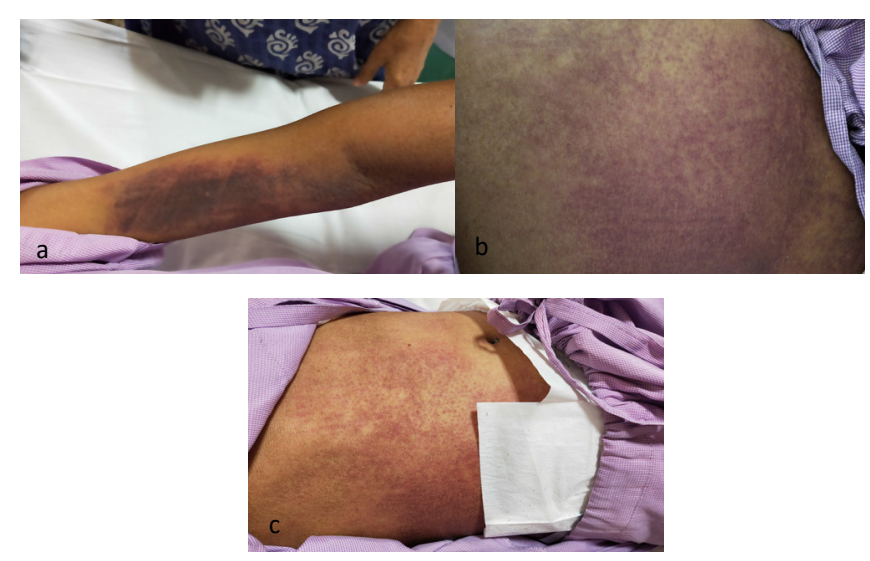

Fig.3: Ecchymosis over (a) upper limb and; (b,c) abdomen.

She was treated with oxygen, inotropic support, empirical antibiotics, methylcobalamine and blood transfusion (pure red blood cells, platelets and fresh frozen plasma). Invasive procedures like bone marrow aspiration and cerebrospinal fluid evaluation could not be done because of thrombocytopenia, coagulopathy, active bleeding and shock. The patient remained in shock and in altered sensorium despite blood transfusion. She was given a trial of hydrocortisone $300 \mathrm{mg}$ intravenous per day in suspicion of adrenal crisis. On day 4, patient's platelet count came down to 6000 and she started bleeding from nose so she was given a trial of high dosage of injection methylprednisolone $1 \mathrm{gm}$ intravenous in view of doubtful history and suspicion of some autoimmune process. Patient's sensorium improved drastically next day and over the next 3 days her sensorium improved completely (GCS 15/15). She was 
shifted to $40 \mathrm{mg}$ oral prednisone which was tapered and stopped over next 7 days. Her haematological profile and liver functions showed improvement and came under normal limits over the next one week. Patient improved in terms of clinical and biochemical profile and was discharged after 3 weeks of admission and asked to follow up in outpatient department.

\section{Discussion}

Idiopathic cytopenia of undetermined significance (ICUS) [2] is an entity of recent times which is used to describe the conditions in which the cause of pancytopenia cannot be determined. The diagnosis of ICUS requires ruling out the secondary causes of pancytopenia including myelodysplastic syndrome and having cytopenia (anemia, leukopenia and thrombocytopenia) for 6 months [2,3]. In our case, duration of disease was unknown as we did not have any past records to label the patient as ICUS. Secondary potential differential diagnosis such as megaloblastic anemia, hematological malignancy, aplastic anemia, infections or splenomegaly. were ruled out. Peripheral pancytopenia can be an early presentation of IMDS (idiopathic myelodysplastic syndrome) and a bone marrow examination is done to rule it out. If it doesn't reveal IMDS then patient is followed up for 6 months and if no evidence of IMDS is found the patient is labelled as ICUS as per international MDS morphology working group [2]. Similar cases of pancytopenia where the cause for pancytopenia could not be established have been reported $[4,5]$. The name "idiopathic fatal pancytopenia" is being given to few case reports where the mechanism of pancytopenia is not clear and patient succumbed to death [6]. In our case, the patient had a life-threatening pancytopenia without any plausible cause so we instituted high dosage of steroids along with the supportive management as a life-saving measure keeping in view the possibility of ICUS/idiopathic fatal pancytopenia and our patient improved drastically. We, in our case could not perform bone marrow biopsy due to low platelets and coagulopathy and after improvement the patient and attendants denied consent for the same. The patient despite having life threatening pancytopenia improved due to steroids, hence she was not labelled as idiopathic fatal pancytopenia. The cause of improvement is not known but the patient remains healthy in 6 months follow up after the illness without any evidence of pancytopenia but due to financial constraints a genetic evaluation has not been done.

\section{Conclusion}

All cases of pancytopenia should be investigated thoroughly and an entity called idiopathic cytopenia of undetermined significance (ICUS) should be considered if no possible causes can be found. We share our experience of instituting steroids in a case of life threatening ICUS which benefitted the patient although the cause and mechanism were unknown.

Contributors: AS: manuscript writing and patient management; AB: manuscript editing and patient management. AS will act as a study guarantor. Both authors approved the final version of this manuscript and are responsible for all aspects of the study.

Funding: None; Competing interests: None stated.

\section{References}

1. Gnanaraj J, Parnes A, Francis CW, Go RS, Takemoto CM, Hashmi SK. Approach to pancytopenia: Diagnostic algorithm for clinical hematologists. Blood Rev. 2018;32(5):361-367.

2. Valent P, Horny HP, Bennett JM, Fonatsch C, Germing $\mathrm{U}$, Greenberg $\mathrm{P}$, et al. Definitions and standards in the diagnosis and treatment of the myelodysplastic syndromes: Consensus statements and report from a working conference. Leukemia Research. 2007;(31):727736.

3. Valent P, Bain BJ, Bennett JM, Wimazal F, Sperr WR, Mufti G, et al. Idiopathic cytopenia of undetermined significance (ICUS) and idiopathic dysplasia of uncertain significance (IDUS), and their distinction from low risk MDS. Leukemia Research. 2012;36:1-5.

4. Lakhey A, Talwar OP, Singh VK, Shiva Raj KC. Clinicohaematological study of pancytopenia. Journal of Pathology of Nepal. 2012;2:207-210.

5. Jha A, Sayami G, Adhikari RC, Patna AD, Jha R. Bone marrow examination in cases of pancytopenia. J Nepal Med Assoc. 2008;47:12-17.

6. Goyal H, Tilak V. Idiopathic Fatal pancytopenia: A case report. J Clin Diagn Res. 2016;10(6):ED09-ED11. 